\title{
RR Lyrae to understand the Galactic halo
}

\author{
Giuliana Fiorentino \\ INAF-Osservatorio Astronomico di Bologna, via Ranzani 1, 40127, Bologna, Italy \\ email: giuliana.fiorentino@oabo.inaf.it
}

\begin{abstract}
We present recent results obtained using old variable RR Lyrae stars on the Galactic halo structure and its connection with nearby dwarf galaxies. We compare the period and periodamplitude distributions for a sizeable sample of fundamental mode RR Lyrae stars (RRab) in dwarf spheroidals ( 1300 stars) with those in the Galactic halo ( 16'000 stars) and globular clusters ( 1000 stars). RRab in dwarfs -as observed today- do not appear to follow the pulsation properties shown by those in the Galactic halo, nor they have the same properties as RRab in globulars. Thanks to the OGLE experiment we extended our comparison to massive metal-rich satellites like the dwarf irregular Large Magellanic Cloud (LMC) and the Sagittarius (Sgr) dwarf spheroidal. These massive and more metal-rich stellar systems likely have contributed to the Galactic halo formation more than classical dwarf spheroidals.

Finally, exploiting the intrinsic nature of RR Lyrae as distance indicators we were able to study the period and period amplitude distributions of RRab within the Halo. It turned out that the inner and the outer Halo do show a difference that may suggest a different formation scenario (in situ vs accreted).
\end{abstract}

Keywords. Galaxy: structure, Galaxy: halo, stars: variables: RR Lyrae

\section{Introduction}

"How did the Galactic halo (Halo) form and evolve?" remains a fundamental open question in astrophysics. Among the different components of our Galaxy the Halo plays a crucial role. The Halo is one of the oldest Galactic component and as a such it is a direct witness of the infancy of the Milky Way (MW). Trapped in its fine and large structure (globular clusters, inner/outer Halo, tidal debris of accreted satellites) there is the key information on how it was formed.

The early suggestion by Searle \& Zinn (1978), based on about 20 GCs, that the outer Halo formed from the aggregation of protogalactic fragments is nowadays supported by 1) theoretical lambda-Cosmological Dark Matter (CDM) simulations of galaxy formation that predict small galaxies form first and then cluster to form larger galaxies, and 2) the observation of stellar streams and merging satellites in the MW and in other galaxies. Currently there are two main open issues concerning the Halo structure and formation:

-Duality of the Halo- Although the Halo was once considered a single component, evidence for its duality is emerging. The existence of an inner and outer (galactocentric distance $\mathrm{d}_{G} \sim 15 \mathrm{Kpc}$ ) component has been recently supported by several works. In particular Carollo et al. $(2007,2010)$ used a large sample $(\sim 16 ’ 000)$ of local stars (within $4 \mathrm{Kpc}$ ) included in recent data release of the Sloan Digital Sky Survey (SDSS, see York et al. 2000). In order to perform an accurate kinematical analysis and to use a simple model for the Halo potential, the authors carefully selected a sample of local stars. Using this sample, they found a kinematic signature of counter rotating and spherically distributed stars that they have attributed to the outer Halo. This is in contraposition with a more flattened and concentrated inner halo component with a zero or slightly 
prograde rotation around the Galactic center. The inner and outer Halo seem also to differ in their chemical compositions. In fact the outer Halo results to be metal poorer $(<[\mathrm{Fe} / \mathrm{H}]>\sim-2.2)$ than the mean peak of the inner Halo $(<[\mathrm{Fe} / \mathrm{H}]>\sim-1.6)$. However, a possible strong bias in the distance determination (up to 50\%) may be the culprit of the "artificial counter-rotating component" (Schonrich et al. 2011). Thus, we still lack a general consensus concerning the Halo large structure;

- The nature of the Halo building blocks -if any- is still a matter of debate. In particular, the question of whether the current dwarf spheroidal satellites (dSphs) of the MW are surviving representatives of the Halo building blocks has been explored in several works but none of them is firmly conclusive (see Tolstoy et al. 2009). Among these, Helmi et al. 2006 found a significant discrepancy between the metallicity distribution function of Halo stars when compared with that observed in dSphs. In particular, the Halo metallicity distribution function shows a very pronounced metal-poor tail which is not present in dSphs. This disagreement seems to be alleviated (but not removed) when selection effect that privileged metal-poor stars are accounted for in the Galactic halo sample (Schorck et al. 2009). Emerging possible candidates as protogalactic fragments are the ultra faint dwarfs that have been recently discovered $\left(\mathrm{L} \lesssim 10^{5} \mathrm{~L}_{\odot}\right.$, e.g. Belokurov et al. 2006). It is worth noting that most of the studies comparing chemical abundances in Halo and dSphs are based on red giant stars (Venn et al. 2004) that suffer the age-metallicity degeneracy. On the other hand the unknown distance (and thus luminosity) of individual red giant Halo stars further complicate a proper comparison. This is why the possibility to compare similar, and preferably old, stellar tracers is crucial in the comparison between the Halo and dSphs. The ideal stellar tracers are old globular clusters (GCs) and field stars (e.g. RR Lyrae, RRL; blue horizontal branch, BHB).

\section{Constraints on the Galactic halo structure}

The use of GCs to constrain the time scale of the Halo formation dates back to half century ago (Eggen et al. 1962, Searle \& Zinn 1978). Stars in GCs have the key advantage to have similar iron abundance (with few exceptions, e.g. $\Omega$ Cen), to be roughly coeval and all at the same distance to us. Thanks to these properties and to the improving accuracy in performing photometry in dense environments, nowadays we are able to estimate very accurate relative ages ( $\delta$ age $\lesssim 1$ Gyr). Marin-Franch et al. 2009 , measured relative ages for a sample of 64 GCs observed with HST optical imaging. They found a dichotomy in the distribution of GCs. A group of young GCs that follows an agemetallicity relation (AMR) and a group of coeval old clusters with an age dispersion of $\sim 5 \%$. These results suggest a scenario where the Halo formed in two steps: 1) a rapid event (duration less than $0.8 \mathrm{Gyr}$ ) that originated the old coeval group; 2) a slow event that may consist of the accretion of nearby satellites and their GCs. A different result has been discussed in Leaman et al. (2013) using a similar sample of 61 GCs. They found that GCs define two parallel AMRs. On the basis of kinematical properties of GCs, this evidence is interpreted in the following way: 1) The metal-poor branch is formed by Halo clusters that were accreted by dwarfs ; 2) The metal-rich branch belongs to the Galactic disk. These two results are controversial and leave the main question of how much Halo was formed from accretion or in situ still open. Here, it is worth noting that also GCs have some drawbacks: i) most of them are within $20 \mathrm{Kpc}$ from the Galactic centre (the Halo extends out to $\sim 100 \mathrm{Kpc}$, Deason et al.2012); ii) they contribute only to few percents of the entire Halo mass $\left(\sim 10^{9} \mathrm{M} \odot\right)$; iii) they are more complex than previously believed in terms of their chemical enrichment histories (Carretta et al. 2009, see also Carretta's 


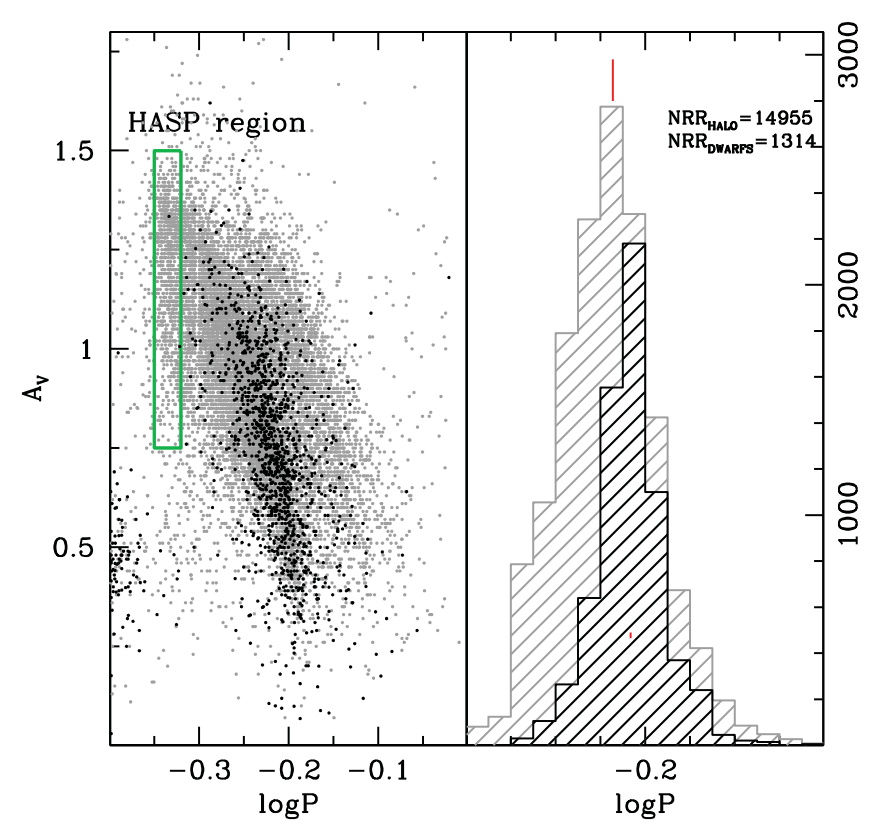

Figure 1. The V-Amplitude vs Period (left) and Period (right) distributions are shown for fundamental RRab stars belonging to the Halo sample (grey) and to the dwarf spheroidal sample (black). The HASP region has been highlighted in green.

review paper, this symposium) and their photometric multiple sequences (e.g., Piotto et al. 2015 and reference therein, see also Piotto's review paper, this symposium). This means that GCs may not be fairly representative of the Halo field stars, thus picturing only part of the story.

Increasing attention has been payed to BHB and RRL stars that can be easily recognised and observed over all across the Halo field. Kinman et al.(2012, and references therein) derived kinematic properties from radial velocities and proper motions of field Halo stars. These authors estimated distances with several different methods for both BHB and RRL stars. Their main conclusion is that a signature for a different kinematics between inner and outer Halo does exist. In particular the outer Halo shows a retrograde motion confirming the result obtained by Carollo and collaborators. Even thought that this is a very carefully done analysis, it is based on a quite limited sample of BHB and RRL stars, i.e. about 100 objects. Gaia will allows us to drastically increase the statistics of the sample where this kind of analysis will be possible returning a full description of the large and fine structure of the Halo. However, waiting for the Gaia data release, much can be learned using the RRLs detected and characterised by the on going photometric and spectroscopic surveys.

\section{New insights from RR Lyrae stars}

There are several good reasons to use RRL stars in this context, they are: 1 ) old ( $\gtrsim$ 10 Gyr) stellar population tracers; 2) excellent distance indicators, thus they can trace the different components of the Galaxy; 3) roughly ubiquitous, they have been observed in several kind of different stellar systems (with enough statistics and the proper metallicity), e.g. globular clusters, classical and ultra-faint dwarf galaxies, Halo, bulge, thick disk ; 4) observationally very easy to pick up and to separate from not (or other kind 
of) variable stars. Hence they are a unique tool to directly compare the ancient stellar population of the Halo at different Galactocentric distance $\left(\mathrm{d}_{G}\right)$ with surrounding stellar systems. Thanks to extensive photometric variability surveys, today we have at disposal robust light curves and hence periods, mean magnitudes and amplitudes for more than 15'000 RR Lyrae belonging to the Halo (QUEST, Zinn et al. 2014; NSVS, Wozniak et al. 2004; ASAS, Szczygiel et al. 2009 and CATALINA, Drake et al. 2013). Using a magnitude vs $[\mathrm{Fe} / \mathrm{H}]$ relation (Cacciari \& Clementini 2003), where the mean metallicity is assumed $[\mathrm{Fe} / \mathrm{H}] \sim-1.6$, one can derive $\mathrm{d}_{G}$ for each individual RRL, they span from $\sim 5$ to $80 \mathrm{Kpc}$. This is the largest, deepest, and most homogeneous catalog at our disposal for the Galactic Halo. Because of time-sampling and completeness problems, the fundamental mode RRLs (hereinafter RRab) stars are the most reliable.

-RRLs and the Halo duality- The period and the period-amplitude distributions, that are reddening and distance independent, can be analysed at varying the $\mathrm{d}_{G}$ using a Kolmogorov-Smirnov (KS) test (see Stetson et al. 2014 and Fiorentino et al. 2015 for details). This predicts a low likelihood that the inner $\left(\mathrm{d}_{G} \lesssim 15 \mathrm{Kpc}\right)$ and the outer $\left(\mathrm{d}_{G} \gtrsim 15 \mathrm{Kpc}\right)$ Halo come from the same parent population. Furthermore, within this catalogue $\sim 3000$ RRab (with $5 \lesssim \mathrm{d}_{G} \lesssim 50 \mathrm{Kpc}$ ) have metallicity estimations from low resolution spectra of $\operatorname{SDSS}(\sigma[\mathrm{Fe} / \mathrm{H}] \sim 0.3)$. They provide the most robust evidence of a metallicity gradient of the ancient population in the Halo (Bono et al. in preparation), similarly to what is found in other massive spiral galaxies. This metallicity gradient varies from $[\mathrm{Fe} / \mathrm{H}] \sim-1.4$ (at $8 \mathrm{Kpc}$ ) to $[\mathrm{Fe} / \mathrm{H}] \sim-1.8$ (at $50 \mathrm{Kpc}$ ). Amplitudes and periods depend on metallicity, hence the KS test prediction of a difference between inner and outer Halo may be only highlighting the presence of the hidden $[\mathrm{Fe} / \mathrm{H}]$-gradient.

-RRLs and the Halo Building blocks-RRLs can also tell us something about the role that classical and ultrafaint dwarf galaxies may have played in the formation of the Halo. Historically, the average properties of RRLs in individual dSphs and UFDs -such as the mean periods of both RRab and RRc stars- are compared to those observed in GCs as representative of the Galactic halo (Smith, Catelan \& Clementini 2009). However, today we have at disposal this unique huge sample of Halo stars that can be directly compared for the first time with the dSph RRL population. To make a proper statistical comparison one can decide to build-up from the literature a homogeneous and complete sample of RRLs in dSphs, this includes Carina, Cetus, Tucana, Leo I, Draco, Ursa Minor and eleven UFDs (see Stetson et al. 2014 and Fiorentino et al. 2015 for the references of the full compilation). It contains more than 1300 RRab stars. This choice is supported by the similarity of the period and period-amplitude distributions of the RRLs in these galaxies. First of all the period distribution of RRab is well peaked around $0.61 \mathrm{~d}$ and it can be well approximated with a gaussian function whereas the Halo distribution is very broader with an average of $\sim 0.58 \mathrm{~d}$ (see Fig.1). Second, a net difference is clear: stars with High Amplitudes and Short Periods (HASP, Av 0.75 mag and $\mathrm{P} \lesssim 0.48 \mathrm{~d}$ ) that are observed in the Halo distribution are not found in any of the considered seventeen dwarfs. The result is solid and indeed it does not change even when the sample of RRab in $\mathrm{dSph}$ is almost doubled including more than 1000 RRab belonging to the massive Fornax dSph (Fiorentino et al. in preparation). Covering a broad range in metallicity $(-2.3 \lesssim[\mathrm{Fe} / \mathrm{H}] \lesssim-1.1$, see Clement et al. 2001$)$, GCs can be used to interpret the HASP evidence. Only GCs hosting more than $35 \mathrm{RRL}$ are accounted for to avoid statistical bias. The metallicity turns out to be the main parameter driving the HASP occurrence, since only GCs more metal-rich than $[\mathrm{Fe} / \mathrm{H}] \sim-1.5$ host RRL in the HASP region. 


\section{Conclusions}

As discussed in Fiorentino et al. (2015), the missing HASP RRab suggest that dSphs and UFDs did not have a metallicity enrichment fast enough to build-up alone the Halo. Scaling the dSphs distribution to the Halo one, an extreme upper limit of $50 \%$ can be estimated to the contribution of current dSphs to the Galactic Halo formation. This is in quite good agreement with recent results of Cosmological simulations (Tissera et al. 2014). Even though the above results rely on rough preliminary estimates, they pose a serious question: "Where does the rest (in fact most) of the Halo mass come from?" There are two main alternatives: 1) from few large and metal-rich stellar systems LMC or Sgr-like (e.g., Zinn et al. 2014); 2) from in situ stellar formation (e.g., Vincenzo et al. 2014). Thanks to the extensive OGLE survey (Soszynski et al. 2009, 2014), more than $\sim 17^{\prime} 000$ and 1600 RRLs in the LMC and Sgr dSph respectively, can be used to investigate the first option. These galaxies both show RRLs in the HASP region. This is reasonable due to the existence of a mass-metallicity relation for the galaxies of the nearby Universe (McConnachie et al. 2012). More quantitatively, a KS test performed on these two galaxies, as compared with the Halo, returns a likelihood of $\sim 10 \%$ for Sgr and few $\%$ for LMC. Although the exceptionally complete LMC sample may affect the statistical test, it is clear that more massive, and thus metal-richer MW satellites may have had a major role in the Halo assembly.

\section{Acknowledgements}

GF is in debt with Monica Tosi and Eline Tolstoy for organizing the meeting "From Dwarfs to Giants" (2013, Sexten, Italy), where the idea of this paper arose from useful discussions. GF has been supported by the FIRB 2013 (grant RBFR13J716).

\section{References}

Carollo, D., et al. 2007, Nature, 450, 1020

Carollo, D., Beers, T. C., Chiba, M., et al. 2010, ApJ, 712, 692

Clement, C. M., et al. 2001, AJ, 122, 2587

Drake, A. J., et al. 2013, ApJ, 763, 32

Eggen, O. J., Lynden-Bell, D., \& Sandage, A. R. 1962, ApJ, 136, 748

Fiorentino, G., Bono, G., Monelli, M., et al. 2015, ApJ (Letters), 798, L12

Helmi, A., et al. 2006, ApJ (Letters), 651, L121

Leaman, R., VandenBerg, D. A., \& Mendel, J. T. 2013, MNRAS , 436, 122

Marín-Franch, A., Aparicio, A., Piotto, G., et al. 2009,ApJ, 694, 1498

McConnachie, A. W. 2012, AJ, 144, 4

Schönrich, R., Asplund, M., \& Casagrande, L. 2011, MNRAS, 415, 3807

Schörck, T., Christlieb, N., Cohen, J. G., et al. 2009, A\&A, 507, 817

Searle, L., \& Zinn, R. 1978, ApJ, 225, 357

Smith, H. A., Catelan, M., \& Clementini, G. 2009, AIP-CP, 1170, 179

Soszyński, I., et al. 2009, AcA, 59, 1

Soszyński, I., Udalski, A., Szymański, M. K., et al. 2014, AcA, 64, 177

Stetson, P. B., Fiorentino, G., Bono, G., Bernard, E. J., Monelli, M., Iannicola, G., Gallart, C., \& Ferraro, I. 2014, PASP, 126, 616

Szczygieł, D. M., Pojmański, G., \& Pilecki, B. 2009, AcA, 59, 137

Tissera, P. B., Beers, T. C., Carollo, D., \& Scannapieco, C. 2014, MNRAS, 439, 3128

Tolstoy, E., Hill, V., \& Tosi, M. 2009, A\&AR, 47, 371

Venn, K. A., Irwin, M., Shetrone, M. D., Tout, C. A., Hill, V., \& Tolstoy, E. 2004, AJ, 128, 1177

Vincenzo, F., Matteucci, F., Vattakunnel, S., \& Lanfranchi, G. A. 2014, MNRAS, 441, 2815 
Vivas, A. K., et al. 2004, AJ, 127, 1158

Woźniak, P. R., Vestrand, W. T., Akerlof, C. W., et al. 2004, AJ, 127, 2436

York, D. G., Adelman, J., Anderson, J. E., Jr., et al. 2000, AJ, 120, 1579

Zinn, R., Horowitz, B., Vivas, A. K., Baltay, C., Ellman, N., Hadjiyska, E., Rabinowitz, D., \& Miller, L. 2014, ApJ, 781, 22 